\title{
Long-term outcome in young women with breast cancer: a population-based study
}

\author{
Hanna Fredholm ${ }^{1,2}$ (D) Kristina Magnusson $^{3} \cdot$ Linda S. Lindström $^{4} \cdot$ \\ Hans Garmo ${ }^{5,6}$ - Sonja Eaker Fält ${ }^{5} \cdot$ Henrik Lindman ${ }^{7} \cdot$ Jonas Bergh $^{8}$. \\ Lars Holmberg $^{5,6} \cdot$ Fredrik Pontén $^{3} \cdot$ Jan Frisell $^{1,2} \cdot$ Irma Fredriksson $^{1,2}$
}

Received: 6 September 2016/Accepted: 7 September 2016/Published online: 13 September 2016

(C) The Author(s) 2016. This article is published with open access at Springerlink.com

\begin{abstract}
Purpose Whether young age at diagnosis of breast cancer is an independent risk factor for death remains controversial, and the question whether young age should be considered in treatment decisions is still to be answered.

Methods From a population-based cohort of 22,017 women with breast cancer, all women $<35$ years $(n=471)$ were compared to a random sample of 700 women aged 35-69 years from the same cohort. Information on patient and tumor characteristics, treatment, and follow-up was collected from the medical records. Tissue microarrays were produced for analysis of classical biomarkers. Breast cancer-specific survival (BCSS), distant disease-free survival (DDFS), and locoregional recurrence-free survival (LRFS) by age were compared using women 50-69 years as reference.

Results At 10 years follow-up, women $<35$ years and 35-39 years had a worse BCSS [age $<35$ years $69 \%$ (HR $2.75,95 \%$ CI 1.93-3.94), age 35-39 years $76 \%$ (HR 2.33, $95 \%$ CI 1.54-3.52), age 40-49 years $84 \%$ (HR 1.53,
\end{abstract}

Electronic supplementary material The online version of this article (doi:10.1007/s10549-016-3983-9) contains supplementary material, which is available to authorized users.

Hanna Fredholm

hanna.fredholm@ki.se

1 Department of Molecular Medicine and Surgery, Karolinska Institutet, Stockholm, Sweden

2 Department of Breast- and Endocrine Surgery, Karolinska University Hospital, 17176 Stockholm, Sweden

3 Science for Life Laboratory, Department of Immunology, Genetics and Pathology, Uppsala University, Uppsala, Sweden

4 Department of Biosciences and Nutrition, Karolinska Institutet, Stockholm, Sweden
$95 \%$ CI 0.97-2.39), and age 50-69 years $89 \%$ (reference)]. The worse BCSS was statistically significant in stages I-IIa and Luminal B tumors. At multivariate analysis age $<35$ years and 35-39 years confined a risk in LRFS (HR 2.13, $95 \%$ CI 1.21-3.76 and HR 1.97, $95 \%$ CI 1.06-3.68) but not in DDFS and BCSS. In the subgroup of women $<40$ years with luminal tumors stage I-IIa, low age remained an independent risk factor also in DDFS (HR $1.87,95 \%$ CI 1.03-3.44).

Conclusion Young women have a high risk of systemic disease even when diagnosed in an early stage. The excess risk of relapse is most pronounced in Luminal B tumors, where low age is an independent prognostic factor of DDFS and LRFS.

Keywords Breast cancer - Young age - Subtype - Luminal B · Early stage $\cdot$ Prognosis · Population-based

\section{Introduction}

Young women with breast cancer have a worse prognosis than middle-aged women [1-7], partly explained by diagnosis at a later stage $[2-4,6,8]$ and by a higher proportion

5 Department of Surgical Sciences, Uppsala University Hospital, Regional Cancer Center, Uppsala University, Uppsala, Sweden

6 Division of Cancer Studies, Faculty of Life Sciences and Medicine, King's College London, London, UK

7 Department of Radiology, Oncology and Radiation Science, Uppsala University Hospital, Uppsala University, Uppsala, Sweden

8 Department of Oncology and Pathology, Cancer Center Karolinska and Karolinska Oncology, Radiumhemmet, Karolinska University Hospital, Karolinska Institutet, Stockholm, Sweden 
of unfavorable tumor characteristics. Young women more often have high grade, hormone receptor-negative, Her2positive tumors, and also more often multifocality, high proliferation, and lymphovascular invasion (LVI) [2, 3, 6, 9-13]. Young women have a higher proportion of intrinsic breast cancer subtypes [14] associated with a worse prognosis: the triple-negative, Her2-positive, and Luminal B subtypes [5, 13, 15-18]. Recently, the prognostic significance of young age has been shown to differ between the different subtypes. Whether young age is an independent prognostic marker for poorer survival even when taking subtype into account remains controversial [5, 10, 15, 18].

In a previous large registry-based cohort study, we found women $<35$ years to have a worse survival than middle-aged women [3]. Stage at diagnosis was a major explanatory factor; however, the excess risk of breast cancer death seen in younger women was only present in early disease, most pronounced in women with small tumors. After correction for stage and tumor characteristics, young age remained an independent risk factor for death.

As it is not likely that young age in itself confers a worse prognosis, but rather this reflects other associations we had not been able to correct for in our registry-based study, we continued with in-depth studies on a large subpopulation from the original cohort. We collected detailed data from the medical records (tumor characteristics, heredity, parity, and treatment), re-evaluated slides (grade, LVI), and collected tumor tissue for TMA providing us with an immunohistochemical (IHC) surrogate of the intrinsic breast subtypes for this population-based cohort study with almost complete and long-term follow-up to study the independent effect of young age on breast cancer-specific survival (BCSS), distant disease-free survival (DDFS), and locoregional recurrence-free survival (LRFS).

\section{Methods}

\section{Study design}

Through the regional breast cancer registries in two of Sweden's six health-care regions, a population-based cohort of 22,017 women with a primary invasive breast cancer diagnosed from 1992 to 2005 at 69 years of age or younger were identified. All women $<35$ years at diagnosis ( $n=471$ ) were compared to random sampled groups of women aged 35-39 years $(n=200), \quad 40-49$ years $(n=200)$, and 50-69 years $(n=300)$ (Flow chart Fig. S1). The sample size was set after power calculations based on the effect sizes from the registry-based study [3]. To reach a power of $80 \%$ at a $95 \%$ significance level, we needed 326 individuals to detect a difference in BCSS and 262 individuals to detect a difference in LRFS.

Information on patient and tumor characteristics, including the treatments given and follow-up until the end of 2012 or until death was collected from the medical records. For women with synchronous bilateral breast cancer, the largest tumor was chosen as the index cancer. Staging was performed using the American Joint Committee on Cancer, 7th edition [19]. The study conforms to the STROBE and REMARK guidelines [20, 21].

\section{Tumor material}

Archival haematoxylin and eosin stained sections and corresponding formalin-fixed and paraffin-embedded tumor blocks were retrieved and histologically reviewed for grade [22] and LVI. Re-sectioning and re-staining were carried out when archival sections were missing. When histological review was not possible, data on grade and LVI were extracted from pathology reports. The presence of multifocality (defined as two or more invasive tumor foci separated by at least $1 \mathrm{~cm}$ ) and ductal carcinoma in situ (DCIS) with extensive growth (defined as $>25 \%$ of the tumor consisting of DCIS with intraductal component also beyond the edge of the invasive tumor) was extracted from the original pathology reports.

TMAs were generated for protein expression profiling using IHC. TMA production, IHC staining, slide scanning, and evaluation of outcome were performed in accordance with strategies and standards used in the Human Protein Atlas project [23, 24]. All patients with tumor material available, 983/1120 (88\%), were included in the set of TMAs. For IHC, the following primary antibodies were used: ER (estrogen receptor) 1:150 (M7047, Dako, Glostrup, Denmark), PR (progesterone receptor) 1:1000 (M3569, Dako), Ki67 1:200 (M7240, Dako), and Her2 1:1000 (A0485, Dako). IHC was performed as previously described [25]. In brief, $4 \mu \mathrm{m}$ sections of the TMA blocks were cut and automated IHC was done using a Lab Vision Autostainer 480 (Thermo Fisher Scientific). The IHCstained and mounted TMA slides were scanned at $\times 20$ magnification with a ScanScope XT system (Aperio Technologies, Vista, USA). The high-resolution digital images of each tissue core were annotated with respect to the outcome of IHC staining. ER was defined as positive when $>1 \%$ of the tumor cell nuclei were positive and PR as positive when $>25 \%$ of the tumor cell nuclei were positive. Ki67 was considered high when $>20 \%$ of the tumor cell nuclei were positive [26, 27]. Her2 was annotated using Her2 ASCO guidelines [28]. Membrane staining intensity of $3+$ was considered positive, while $2+$ was further verified through chromogenic in situ hybridization (CISH) to determine Her2-gene amplification [28-30]. 
CISH was performed on an automated Ventana BenchMark ULTRA IHC/ISH Staining Module (Ventana Medical Systems, Inc Tuscon, AZ, USA) using the INFORM HER2 Dual ISH DNA Probe Cocktail. CISH-stained slides were examined under the microscope and the amount of positive Her2 signals scored in tumor cell nuclei. The outcome was scored as Her2 amplified ( $>6$ dots or clusters of positive signal) or non-Her2 amplified ( $\leq 6$ dots per nuclei).

To define the intrinsic breast cancer subtypes, we used surrogate definitions based on central IHC re-evaluation of ER, PR, Ki67, and Her2 according to the St Gallen consensus statement [27]. Luminal A was defined as ER+, $\mathrm{PR}+$, Her2-, and Ki67 low, Luminal $\mathrm{B}$ as ER+, PR+, Her2-, and Ki67 high or ER+, PR -, Her2-, and any Ki67, Luminal-Her2 as ER+ and Her2+, any PR or Ki67, Her2positive (non-luminal) as $\mathrm{ER}-, \mathrm{PR}-$ and Her2+, any Ki67 and triple-negative as ER-, PR- and Her2-, any Ki67.

\section{Statistical analysis}

Endpoints were BCSS, DDFS, and LRFS. BCSS was calculated using time from diagnosis to death from breast cancer censoring for end of follow-up. DDFS was estimated using time from diagnosis to distant recurrence or death from breast cancer, whichever came first censoring for the end of follow-up. LRFS was calculated using time from diagnosis to locoregional recurrence as first event. Kaplan-Meier curves were used to estimate survival time [31] as death from other causes than breast cancer was uncommon in this population. Survival curves were compared using log-rank test [32]. Cox proportional-hazards models were used to estimate the univariate and multivariate hazard ratios (HR) and $95 \%$ confidence intervals (95\% CI) [33]. All statistical tests were two-sided and $p$ values $<0.05$ were deemed significant. All calculations were performed using IBM SPSS Statistics v22.0 (SPSS Inc. Illinois, USA).

\section{Results}

\section{Population characteristics}

Data on patient and tumor characteristics divided by age group are shown in Table 1 . Women $<35$ years had larger tumors and more often involved lymph nodes than women aged 50-69 years. Fewer women $<35$ years presented with stage I disease. Women $<35$ years more often had tumors that were grade III, hormone receptor negative, Her2-positive, and high Ki67. This translates to a lower proportion of the luminal subtypes and a higher proportion of the triple-negative and Her2-positive subtypes among younger women. Multifocal disease, LVI, and the presence of extensive DCIS were more common in women $<35$ years. Altogether, characteristics in women 35-39 years were similar to those in women $<35$ years, whereas the characteristics in women 40-49 years group together well with those aged 50-69 years.

Treatment was performed according to the national guidelines for each time period, closely following international practice. Data on treatment by age group are shown in Table 2 and time trends of systemic treatment in relation to age, tumor size, lymph node status, grade, and subtype are presented in Fig. S2.

Median follow-up time was 10 years (range 0-20). In the group aged $<35$ years, 90 of 445 had a locoregional recurrence as first event. The corresponding figures were for women 35-39 years 37 of 190, 40-49 years 27 of 192, and 50-69 years 22 of 293. Distant disease occurred in 169 of 445 women <35 years, in 59 of 190 women aged 35-39 years, in 47 of 192 women aged 40-49 years, and in 42 of 293 women aged 50-69 years.

\section{Univariate analysis}

Univariate analyses of risk factors for breast cancer death stratified by age are shown in Table 3. The increased risk of breast cancer death in young versus middle-aged women was significant during the earlier part of the studied period and mainly noted in tumors with favorable characteristics, namely: small tumor size, low grade, Her2-negativity, and no LVI.

At 10-year follow-up, the BCSS was for women $<35$ years $69 \%$ (HR 2.75, $95 \%$ CI 1.93-3.94), for women 35-39 years $76 \%$ (HR 2.33, $95 \%$ CI 1.54-3.52), for women 40-49 years $84 \% \quad(\mathrm{HR} \quad 1.53,95 \% \quad \mathrm{CI}$ 0.97-2.39), and women 50-69 years $89 \% \quad(\mathrm{HR}=1.00$ reference) (Fig. 1).

Figure 2 shows BCSS by tumor characteristics and age. Women aged $<40$ years had a statistically significantly worse survival than women $\geq 40$ years in stages I and IIa (HR 3.03, $95 \%$ CI 1.65-5.57 and HR 2.08, $95 \%$ CI 1.16-3.74), irrespective of tumor grade (grade I; HR 12.25, $95 \%$ CI 1.35-111.17, grade II; HR 1.82, $95 \%$ CI 1.15-2.87 and grade III; HR 1.50, $95 \%$ CI 1.01-2.23), and in the Luminal B subtype $(\mathrm{HR}=1.79,95 \% \mathrm{CI}=1.15$ $2.78)$. In women $<40$ years, the best survival was seen in those with Luminal A tumors (10-year BCSS $92 \%$ ) while it was markedly worse in the other subtypes (Luminal B $75 \%$, Her2-positive $68 \%$ (in this analysis Luminal-Her2 and Her2-positive combined), and triple-negative $67 \%$ ).

\section{Multivariate analysis}

In the multivariate analysis (Table 4), successively correcting for year of diagnosis, stage at diagnosis, detection 
Table 1 Patient- and tumor characteristics for women with primary breast cancer stage I-III diagnosed 1992-2005, by age at diagnosis $(N=1120)$

\begin{tabular}{|c|c|c|c|c|c|c|c|c|}
\hline & \multicolumn{2}{|c|}{$<35$ years } & \multicolumn{2}{|c|}{ 35-39 years } & \multicolumn{2}{|c|}{ 40-49 years } & \multicolumn{2}{|c|}{$50-69$ years } \\
\hline & \multicolumn{2}{|c|}{$n=445$} & \multicolumn{2}{|c|}{$n=190$} & \multicolumn{2}{|c|}{$n=192$} & \multicolumn{2}{|c|}{$n=293$} \\
\hline & No. & $(\%)$ & No. & $(\%)$ & No. & $(\%)$ & No. & $(\%)$ \\
\hline \multicolumn{9}{|l|}{ Year of diagnosis } \\
\hline 1992-1997 & 169 & $(38.0)$ & 82 & $(43.2)$ & 86 & $(44.8)$ & 89 & $(30.4)$ \\
\hline 1998-2002 & 175 & $(39.3)$ & 61 & $(32.1)$ & 62 & $(32.3)$ & 132 & $(45.1)$ \\
\hline 2003-2005 & 101 & $(22.7)$ & 47 & $(24.7)$ & 44 & $(22.9)$ & 72 & $(24.6)$ \\
\hline Detection by screening & 6 & $(1.3)$ & 5 & (2.6) & 45 & $(23.4)$ & 167 & $(57.0)$ \\
\hline \multicolumn{9}{|l|}{ Heredity $^{\mathrm{a}}$} \\
\hline Any heredity & 187 & $(42.0)$ & 73 & $(38.4)$ & 59 & $(30.7)$ & 80 & $(27.3)$ \\
\hline$\geq 1$ first grade relative & 81 & $(18.2)$ & 37 & $(19.5)$ & 25 & $(13.0)$ & 50 & $(17.1)$ \\
\hline \multicolumn{9}{|l|}{ Tumor size } \\
\hline $1-10 \mathrm{~mm}$ & 67 & $(15.1)$ & 27 & $(14.2)$ & 35 & $(18.2)$ & 77 & $(26.3)$ \\
\hline $11-20 \mathrm{~mm}$ & 148 & $(33.3)$ & 72 & $(37.9)$ & 80 & $(41.7)$ & 136 & $(46.4)$ \\
\hline $21-50 \mathrm{~mm}$ & 189 & $(42.5)$ & 72 & $(37.9)$ & 70 & $(36.5)$ & 67 & $(22.9)$ \\
\hline$>51 \mathrm{~mm}$ & 38 & $(8.5)$ & 16 & (8.4) & 6 & (3.1) & 11 & (3.8) \\
\hline Missing & 3 & $(0.7)$ & 3 & (1.6) & 1 & $(0.5)$ & 2 & $(0.7)$ \\
\hline \multicolumn{9}{|l|}{ Lymph node status } \\
\hline Node neg & 227 & (51.0) & 90 & $(47.4)$ & 116 & $(60.4)$ & 214 & $(73.0)$ \\
\hline $1-3$ nodes pos & 126 & $(28.3)$ & 68 & $(35.8)$ & 47 & $(24.5)$ & 57 & $(19.5)$ \\
\hline$>4$ nodes pos & 92 & (20.7) & 32 & $(16.8)$ & 29 & $(15.1)$ & 22 & $(7.5)$ \\
\hline \multicolumn{9}{|l|}{ Stage } \\
\hline I & 14 & (32.4) & 63 & $(33.2)$ & 87 & $(45.3)$ & 172 & $(58.7)$ \\
\hline IIa & 126 & $(28.3)$ & 50 & $(26.3)$ & 44 & $(22.9)$ & 74 & $(25.3)$ \\
\hline $\mathrm{IIb}$ & 71 & $(16.0)$ & 35 & $(18.4)$ & 28 & $(14.6)$ & 21 & $(7.2)$ \\
\hline III & 103 & $(23.1)$ & 40 & $(21.1)$ & 33 & $(17.2)$ & 26 & (8.9) \\
\hline Unstaged & 1 & $(0.2)$ & 2 & $(1.1)$ & 0 & & 0 & \\
\hline \multicolumn{9}{|l|}{ Grade (Elston) } \\
\hline I & 21 & $(5.3)$ & 21 & $(12.7)$ & 31 & $(17.9)$ & 75 & $(27.3)$ \\
\hline II & 140 & $(35.6)$ & 63 & $(38.2)$ & 79 & $(45.7)$ & 131 & $(47.6)$ \\
\hline III & 232 & $(59.0)$ & 81 & $(49.1)$ & 63 & $(36.4)$ & 69 & $(25.1)$ \\
\hline Missing & 52 & & 25 & & 19 & & 18 & \\
\hline \multicolumn{9}{|l|}{ Estrogen receptor ${ }^{\mathrm{b}}$} \\
\hline Pos & 208 & $(47.2)$ & 122 & $(64.9)$ & 146 & $(77.7)$ & 225 & $(78.1)$ \\
\hline Neg & 233 & $(52.8)$ & 66 & $(35.1)$ & 42 & $(22.3)$ & 63 & $(21.9)$ \\
\hline Missing & 4 & & 2 & & 4 & & 5 & \\
\hline \multicolumn{9}{|l|}{ Progesterone receptor ${ }^{\mathrm{b}}$} \\
\hline Pos & 155 & $(35.4)$ & 85 & $(45.2)$ & 114 & $(60.6)$ & 145 & $(51.1)$ \\
\hline Neg & 283 & $(64.6)$ & 103 & $(54.8)$ & 74 & $(39.4)$ & 139 & $(48.9)$ \\
\hline Missing & 7 & & 2 & & 4 & & 9 & \\
\hline \multicolumn{9}{|l|}{ Ki-67 (\%) } \\
\hline Low $\leq 20$ & 70 & $(18.8)$ & 42 & $(26.9)$ & 67 & $(40.1)$ & 127 & $(51.2)$ \\
\hline High $>20$ & 302 & $(81.2)$ & 114 & $(73.1)$ & 100 & $(59.9)$ & 121 & $(48.8)$ \\
\hline Missing & 73 & & 34 & & 25 & & 45 & \\
\hline \multicolumn{9}{|l|}{ Her2 } \\
\hline Neg & 296 & (79.6) & 127 & $(81.4)$ & 150 & $(90.4)$ & 225 & (91.8) \\
\hline Pos & 76 & (20.4) & 29 & $(18.6)$ & 16 & $(9.6)$ & 20 & $(8.2)$ \\
\hline Missing & 73 & & 34 & & 26 & & 48 & \\
\hline
\end{tabular}


Table 1 continued

\begin{tabular}{|c|c|c|c|c|c|c|c|c|}
\hline & \multicolumn{2}{|c|}{$<35$ years } & \multicolumn{2}{|c|}{ 35-39 years } & \multicolumn{2}{|c|}{ 40-49 years } & \multicolumn{2}{|c|}{$50-69$ years } \\
\hline & \multicolumn{2}{|c|}{$n=445$} & \multicolumn{2}{|c|}{$n=190$} & \multicolumn{2}{|c|}{$n=192$} & \multicolumn{2}{|c|}{$n=293$} \\
\hline & No. & $(\%)$ & No. & $(\%)$ & No. & $(\%)$ & No. & $(\%)$ \\
\hline \multicolumn{9}{|l|}{ Subtype } \\
\hline Luminal A & 27 & (7.7) & 23 & $(15.1)$ & 40 & $(25.8)$ & 59 & $(25.9)$ \\
\hline Luminal B & 132 & $(37.5)$ & 66 & $(43.4)$ & 80 & $(51.6)$ & 117 & $(51.3)$ \\
\hline Luminal-Her2 & 35 & $(9.9)$ & 16 & $(10.5)$ & 7 & $(4.5)$ & 10 & $(4.4)$ \\
\hline Her2-positive & 40 & $(11.4)$ & 13 & (8.6) & 8 & $(5.2)$ & 9 & $(3.9)$ \\
\hline Triple-negative & 118 & $(33.5)$ & 34 & $(22.4)$ & 20 & $(12.9)$ & 33 & $(14.5)$ \\
\hline Unclassified & 93 & & 38 & & 37 & & 65 & \\
\hline \multicolumn{9}{|l|}{ Presence of: } \\
\hline $\mathrm{LVI}^{\mathrm{b}}$ & 139 & $(31.2)$ & 43 & (22.6) & 39 & (20.3) & 32 & $(10.9)$ \\
\hline Invasive multifocality & 96 & $(21.6)$ & 39 & $(20.5)$ & 35 & $(18.2)$ & 46 & $(15.7)$ \\
\hline Extensive DCIS & 92 & $(20.7)$ & 43 & $(22.6)$ & 30 & (15.6) & 37 & (12.6) \\
\hline
\end{tabular}

mode, grade, subtype, and systemic treatment, young age $(<35$ years and $35-39$ years) was an independent risk factor in LRFS (HR 2.13, $95 \%$ CI 1.21-3.76 and HR 1.97, $95 \%$ CI 1.06-3.68) but not in DDFS or BCSS.

To focus on the subpopulation of women where the survival analyses indicated substantial differences between women aged $<40$ and $\geq 40$ years (Luminal Her- 2 negative breast cancer stage I-IIa), we performed a separate multivariate analysis (Fig. 3). Age $<40$ years was a statistically significant independent risk factor in DDFS (HR 1.87, $95 \%$ CI 1.03-3.44) and in LRFS (HR 4.10, $95 \%$ CI 2.20-7.66), but not in BCSS (HR 1.47, $95 \%$ CI $0.72-3.02)$.

\section{Discussion}

This population-based cohort study included 1120 women with breast cancer stage I-III of which 445 were $<35$ years at diagnosis with a median follow-up of 10 years. Women aged $<35$ years and 35-39 years had more advanced stage at diagnosis and a higher proportion of Her2-positive and triple-negative subtypes and less common Luminal A subtype. Women $<35$ years and 35-39 years received more intense treatment reflecting their stage and subtype distribution. Women $<40$ years had a worse BCSS compared to women $\geq 40$ years in stage I and IIa, in all tumor grades and in the Luminal B subtype. At multivariate analysis, age remained an independent risk factor in LRFS but not significantly in BCSS or DDFS. In women with luminal early-stage disease, young age was an independent risk factor also of DDFS.
Treatment was given according to national guidelines and best international practice at that time. The number of women $<35$ years and 35-39 years is large, with detailed data on patient, tumor, and treatment characteristics and follow-up extracted from medical records. The long-term follow-up is nearing completion.

In a central pathology review, we re-evaluated grade and LVI and re-analyzed prognostic markers with modern methods at one single laboratory. Using IHC methods to separate Luminal A from Luminal B tumors has limitations [34]. In this study, we performed new IHC-analyses on archival material to avoid the effects of low intra- and inter-laboratory reproducibility and different antibodies for testing. To validate our results with regard to the arbitrarily set cutoffs, we performed a sensitivity analysis using alternative subtype definitions; grade instead of Ki67, Ki67 cutoff $14 \%$, ER-positive cutoff $>10 \%$ stained nuclei, which did not change the results.

During the study period of 14 years, treatment regimes have changed, and the time trends have not been the same in the compared age groups. More intense treatment was offered to young women and modern regimes were introduced earlier, which might have led to an underestimation of age-related survival differences in the multivariate analyses.

Many studies have shown young women with breast cancer to have a worse prognosis compared to their older counterparts. Our findings demonstrate that the differences in BCSS between age groups diminished over time, and lost significance during the last part of the studied period. In a recent Canadian study, outcomes for young breast cancer patients across two time periods were compared to 
Table 2 Given treatment for women with primary breast cancer stage I-III diagnosed 1992-2005, by age at diagnosis $(N=1120)$

\begin{tabular}{|c|c|c|c|c|c|c|c|c|}
\hline & \multirow{2}{*}{\multicolumn{2}{|c|}{$\begin{array}{l}<35 \text { years } \\
n=445\end{array}$}} & \multirow{2}{*}{\multicolumn{2}{|c|}{$\begin{array}{l}35-39 \text { years } \\
n=190\end{array}$}} & \multirow{2}{*}{\multicolumn{2}{|c|}{$\frac{40-49 \text { years }}{n=192}$}} & \multirow{2}{*}{\multicolumn{2}{|c|}{$\begin{array}{l}50-69 \text { years } \\
n=293\end{array}$}} \\
\hline & & & & & & & & \\
\hline & No. & $(\%)$ & No. & $(\%)$ & No. & $(\%)$ & No. & $(\%)$ \\
\hline \multicolumn{9}{|l|}{ Breast surgery } \\
\hline BCS & 206 & $(46.3)$ & 94 & $(49.5)$ & 117 & $(60.9)$ & 194 & $(66.2)$ \\
\hline Mastectomy & 239 & $(53.7)$ & 94 & $(49.5)$ & 74 & $(38.5)$ & 99 & $(33.8)$ \\
\hline No surgery & 0 & & 2 & $(1.1)$ & 1 & $(0.5)$ & 0 & \\
\hline \multicolumn{9}{|l|}{ Chemotherapy } \\
\hline No & 109 & $(24.5)$ & 48 & $(25.3)$ & 103 & $(53.6)$ & 204 & $(69.6)$ \\
\hline Yes & 336 & $(75.5)$ & 142 & $(74.7)$ & 89 & $(46.4)$ & 89 & $(30.4)$ \\
\hline $\mathrm{CMF}$ & 78 & $(23.2)$ & 45 & $(31.7)$ & 27 & $(30.3)$ & 24 & $(27.0)$ \\
\hline FEC & 208 & $(61.9)$ & 82 & $(57.7)$ & 54 & $(60.7)$ & 60 & $(67.4)$ \\
\hline Taxanes & 47 & $(14.0)$ & 15 & $(10.6)$ & 6 & $(6.7)$ & 5 & $(5.6)$ \\
\hline Other & 3 & $(0.9)$ & 0 & & 2 & $(2.2)$ & 0 & \\
\hline Proportion neoadjuvant & 76 & $(17.1)$ & 29 & $(15.3)$ & 13 & $(6.8)$ & 8 & $(2.7)$ \\
\hline Chemotherapy when $\mathrm{N}+$ & 214 & $(98.2)$ & 95 & $(95.0)$ & 72 & $(94.7)$ & 61 & $(77.2)$ \\
\hline Chemotherapy when hormone rec $\operatorname{pos}_{a}$ & 169 & $(67.6)$ & 85 & $(65.9)$ & 65 & $(41.7)$ & 59 & $(25.0)$ \\
\hline \multicolumn{9}{|l|}{ Trastuzumab } \\
\hline Yes & 18 & $(4.0)$ & 5 & $(2.6)$ & 3 & (1.6) & 4 & $(1.4)$ \\
\hline No & 427 & $(96.0)$ & 185 & $(97.4)$ & 189 & $(98.4)$ & 289 & $(98.6)$ \\
\hline \multicolumn{9}{|l|}{ Radiotherapy } \\
\hline Yes & 358 & $(80.4)$ & 149 & $(78.4)$ & 160 & $(83.3)$ & 231 & $(78.8)$ \\
\hline No & 87 & $(19.6)$ & 41 & $(21.6)$ & 32 & $(16.7)$ & 62 & $(21.2)$ \\
\hline Breast radiation when $\mathrm{BCS}$ & 196 & $(95.1)$ & 92 & $(97.9)$ & 113 & $(96.6)$ & 187 & $(96.4)$ \\
\hline Chest wall radiation when mastectomy & 162 & $(67.8)$ & 57 & $(60.6)$ & 46 & $(62.2)$ & 43 & $(43.4)$ \\
\hline Axillary radiation when $\mathrm{N}+$ & 168 & $(77.1)$ & 68 & $(68.0)$ & 49 & $(64.5)$ & 58 & $(73.4)$ \\
\hline \multicolumn{9}{|l|}{ Endocrine therapy } \\
\hline Yes & 208 & $(46.7)$ & 90 & $(47.4)$ & 109 & $(56.8)$ & 190 & $(64.8)$ \\
\hline No & 237 & $(53.3)$ & 100 & $(52.6)$ & 82 & $(42.7)$ & 102 & $(34.8)$ \\
\hline Missing & 0 & & 0 & & 1 & $(0.5)$ & 1 & $(0.3)$ \\
\hline Endocrine therapy ${ }^{\mathrm{b}}$ when hormone rec pos ${ }^{\mathrm{a}}$ & 176 & $(70.4)$ & 80 & $(62.0)$ & 97 & $(62.2)$ & 177 & $(75.0)$ \\
\hline Ovarian suppression when hormone rec pos ${ }^{\mathrm{a}}$ & 79 & (31.9) & 28 & $(22.0)$ & 17 & (11.1) & 4 & (1.7) \\
\hline
\end{tabular}

$B C S$ breast conserving surgery, $C M F$ cyclophosphamide, methotrexate, 5-fluorouracil, FEC 5-fluorouracil, epirubicin, cyclophosphamide, $N+$ lymph node positive

${ }^{a}$ Hormone receptor positive defined as either ER pos or PR pos

${ }^{\mathrm{b}}$ Endocrine therapy including ovarian suppression determine whether the poor prognosis persists in the context of modern adjuvant therapies. There was an improvement in breast cancer outcome over time for all subgroups, but age $<40$ continued to predict inferior survival despite modern therapies [18]. Published data from The Surveillance, Epidemiology, and End Results program showed improved outcomes for young women with breast cancer over time, however restricted only to women with ER-positive disease [35].

The difference in prognosis between age groups has consistently been reported to be particularly evident in young women with ER-positive tumors [36-40]. More recently, the prognostic significance of young age has been shown to be most prominent in the Luminal B subtype $[5,12,15,18,41]$ even though some reports have indicated an increased risk compared with older women also among young with triple-negative [15, 16] and Her2-positive subtypes [42, 43]. In the present study, women aged $<40$ had a significantly worse survival only in the Luminal B subtype. Thus, the effect of age seems to vary within tumor subtypes.

Morrison et al. found Luminal B tumors among young women to demonstrate more aggressive features, with significantly lower ER and PR levels, higher Ki67, and p53 overexpression, than in older women with the same subtype. The high proliferation and p53 level, coupled with 
Table 3 Univariate analysis of risk factors for breast cancer death by age for women with stage I-III breast cancer diagnosed 1992-2005 $(N=1120)$

\begin{tabular}{|c|c|c|c|c|c|c|c|}
\hline \multirow[b]{3}{*}{ Unadjusted } & \multicolumn{2}{|c|}{$<35$ years } & \multicolumn{2}{|c|}{ 35-39 years } & \multicolumn{2}{|c|}{ 40-49 years } & \multirow{2}{*}{$\begin{array}{l}50-69 \text { years } \\
n=293\end{array}$} \\
\hline & \multicolumn{2}{|c|}{$n=445$} & \multicolumn{2}{|c|}{$n=190$} & \multicolumn{2}{|c|}{$n=192$} & \\
\hline & 2.75 & $(1.93-3.94)$ & 2.33 & $(1.54-3.52)$ & 1.53 & $(0.97-2.39)$ & \\
\hline \multicolumn{8}{|l|}{ Year of diagnosis } \\
\hline 1992-1997 & 2.18 & $(1.31-3.63)$ & 2.04 & $(1.16-3.59)$ & 1.28 & $(0.70-2.35)$ & \\
\hline 1998-2002 & 4.02 & $(2.16-7.50)$ & 2.93 & $(1.37-6.27)$ & 1.80 & $(0.78-4.16)$ & \\
\hline 2003-2005 & 1.90 & $(0.79-4.54)$ & 1.33 & $(0.45-3.95)$ & 1.17 & $(0.37-3.68)$ & \\
\hline Non screening detection & 1.80 & $(1.17-2.78)$ & 1.54 & $(0.95-2.49)$ & 1.16 & $(0.69-1.96)$ & \\
\hline Positive heredity & 1.47 & $(0.84-2.58)$ & 1.41 & $(0.73-2.73)$ & 1.15 & $(0.56-2.35)$ & \\
\hline \multicolumn{8}{|l|}{ Tumor size (mm) } \\
\hline$\leq 20$ & 3.42 & $(1.91-6.12)$ & 3.19 & $(1.65-6.20)$ & 1.75 & $(0.85-3.63)$ & \\
\hline $21-50$ & 1.60 & $(0.93-2.76)$ & 1.49 & $(0.79-2.78)$ & 1.24 & $(0.65-2.37)$ & \\
\hline$\geq 51$ & 1.26 & $(0.51-3.11)$ & 0.55 & $(0.18-1.70)$ & 0.23 & $(0.03-1.93)$ & \\
\hline \multicolumn{8}{|l|}{ Lymph node status } \\
\hline Negative & 2.10 & $(1.22-3.62)$ & 2.30 & $(1.21-4.39)$ & 0.63 & $(0.26-1.49)$ & \\
\hline 1-3 nodes positive & 2.63 & $(1.33-5.19)$ & 1.90 & $(0.90-4.00)$ & 1.20 & $(0.50-2.89)$ & \\
\hline$\geq 4$ nodes positive & 1.41 & $(0.70-2.87)$ & 1.05 & $(0.45-2.41)$ & 2.05 & $(0.94-4.47)$ & \\
\hline \multicolumn{8}{|l|}{ Stage } \\
\hline I & 3.07 & $(1.41-6.68)$ & 3.75 & $(1.58-8.90)$ & 1.24 & $(0.44-3.48)$ & \\
\hline IIa & 1.55 & $(0.82-2.94)$ & 1.24 & $(0.56-2.78)$ & 0.24 & $(0.05-1.06)$ & \\
\hline IIb & 1.71 & $(0.71-4.08)$ & 1.20 & $(0.45-3.15)$ & 0.83 & $(0.29-2.39)$ & \\
\hline III & 1.42 & $(0.73-2.80)$ & 1.04 & $(0.48-2.27)$ & 1.94 & $(0.92-4.10)$ & \\
\hline \multicolumn{8}{|l|}{ Grade } \\
\hline $\mathrm{I}-\mathrm{II}$ & 3.25 & $(1.81-5.81)$ & 2.38 & $(1.18-4.81)$ & 1.64 & $(0.80-3.36)$ & \\
\hline III & 1.46 & $(0.87-2.47)$ & 1.37 & $(0.75-2.51)$ & 0.91 & $(0.46-1.83)$ & \\
\hline \multicolumn{8}{|l|}{ Estrogen receptor } \\
\hline Positive & 2.89 & $(1.85-4.53)$ & 2.28 & $(1.36-3.85)$ & 1.36 & $(0.78-2.39)$ & \\
\hline Negative & 1.91 & $(1.03-3.52)$ & 1.85 & $(0.91-3.73)$ & 1.59 & $(0.73-3.49)$ & \\
\hline Progesterone receptor & & & & & & & 1.00 (ref.) \\
\hline Positive & 2.77 & $(1.53-5.01)$ & 2.45 & $(1.26-4.79)$ & 1.10 & $(0.52-2.31)$ & \\
\hline Negative & 2.37 & $(1.51-3.73)$ & 2.04 & $(1.20-3.48)$ & 1.91 & $(1.07-3.41)$ & \\
\hline \multicolumn{8}{|l|}{ Ki67 (\%) } \\
\hline Low $\leq 20$ & 3.15 & $(1.49-6.67)$ & 1.33 & $(0.46-3.82)$ & 1.72 & $(0.73-4.06)$ & \\
\hline High $\geq 21$ & 1.70 & $(1.08-2.68)$ & 1.65 & $(0.98-2.78)$ & 1.09 & $(0.61-1.96)$ & \\
\hline \multicolumn{8}{|l|}{ Her2 } \\
\hline Negative & 2.36 & $(1.55-3.60)$ & 2.15 & $(1.31-3.53)$ & 1.27 & $(0.74-2.17)$ & \\
\hline Positive & 1.45 & $(0.56-3.74)$ & 0.78 & $(0.25-2.46)$ & 1.38 & $(0.42-4.52)$ & \\
\hline \multicolumn{8}{|l|}{ Subtype } \\
\hline Luminal A & 1.84 & $(0.49-6.86)$ & 0.55 & $(0.06-4.67)$ & 0.29 & $(0.03-2.44)$ & \\
\hline Luminal B & 2.30 & $(1.27-4.19)$ & 2.30 & $(1.18-4.49)$ & 1.64 & $(0.82-3.28)$ & \\
\hline Luminal-Her2 & 0.84 & $(0.23-3.02)$ & 0.75 & $(0.18-3.16)$ & 0.75 & $(0.13-4.50)$ & \\
\hline Her2-positive & 1.77 & $(0.41-7.67)$ & 0.56 & $(0.08-3.98)$ & 2.20 & $(0.40-12.03)$ & \\
\hline Triple-negative & 1.26 & $(0.61-2.61)$ & 1.35 & $(0.57-3.21)$ & 1.09 & $(0.39-3.07)$ & \\
\hline \multicolumn{8}{|l|}{ Lymphovascular invasion } \\
\hline No & 2.66 & $(1.74-4.06)$ & 2.12 & $(1.29-3.49)$ & 1.14 & $(0.64-2.01)$ & \\
\hline Yes & 1.56 & $(0.77-3.15)$ & 1.57 & $(0.71-3.47)$ & 1.60 & $(0.71-3.60)$ & \\
\hline \multicolumn{8}{|l|}{ Invasive multifocality } \\
\hline No & 3.08 & $(2.04-4.63)$ & 2.18 & $(1.33-3.55)$ & 1.62 & $(0.97-2.71)$ & \\
\hline
\end{tabular}


Table 3 continued

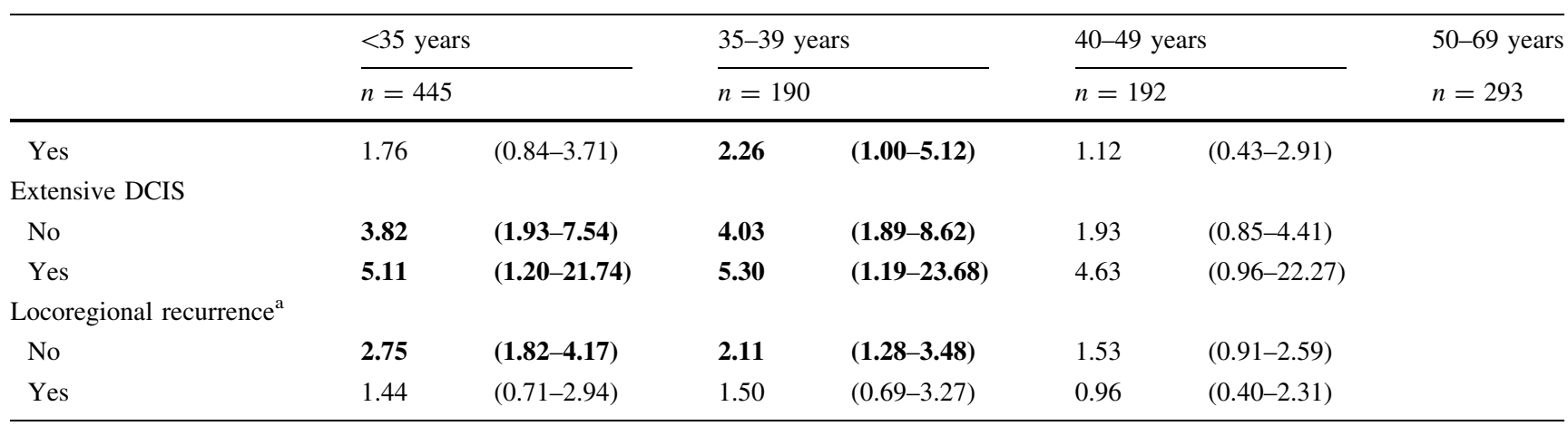

Hazard ratio (95\% confidence interval) for risk of breast cancer death according to age and one additional risk factor

Bold values indicate statistical significance at the $p<0.05$ level

${ }^{\text {a }}$ Locoregional recurrence as first event

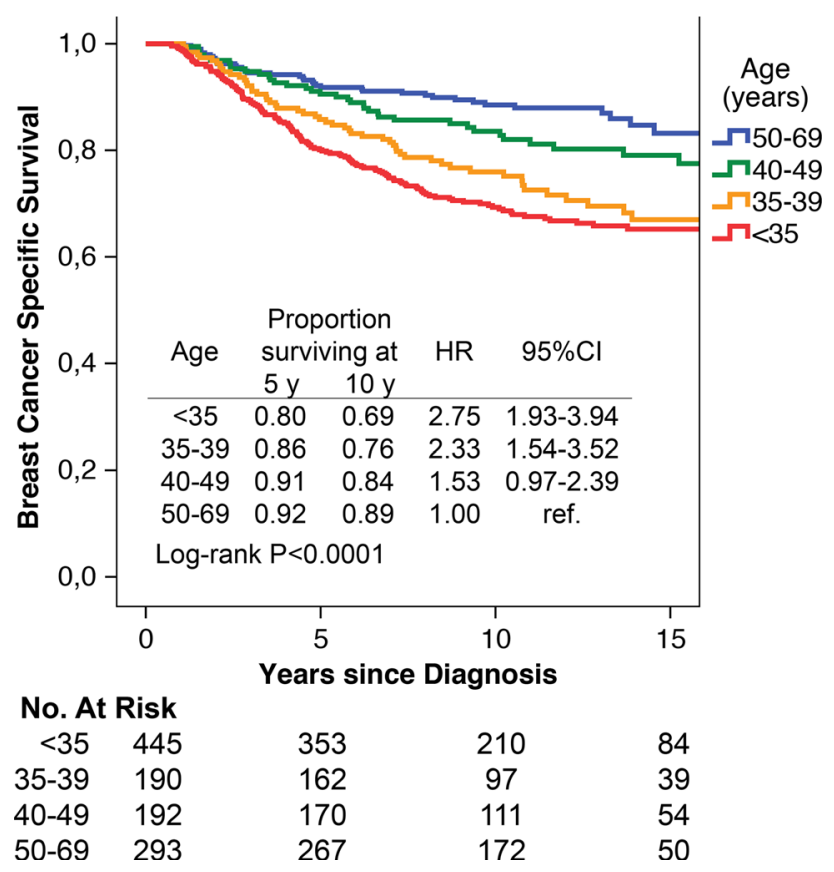

Fig. 1 Breast cancer-specific survival by age in a population-based cohort of 1120 women with primary breast cancer stage I-III diagnosed 1992-2005 divided by age <35 years, 35-39 years, 40-49 years, and 50-69 years. Proportion of women surviving at 5, 10 , and 15 years from diagnosis. Hazard ratios (HR) of breast cancer death are given with their $95 \%$ confidence intervals (95\% CI). Survival curves are compared by log-rank test

low ER and PR expression in young women, suggests that these tumors may originate from less-differentiated luminal cells [13].

Using genomic expression analysis, Azim and colleagues could, even after adjustment for subtype, observe remaining genetic differences by age with enrichment of processes related to immature mammary epithelial cells, growth factor signaling, and down-regulation of apoptosis- related genes [5]. Johnson et al. studied age-related gene expression differences within and across breast cancer subtypes. After adjustment for subtype, four key genes for proliferation, invasion, and metastasis persisted, some of which predicted inferior disease-free survival in younger women [43]. Also Liao et al. demonstrated unique genomic signatures differentiating premenopausal breast cancer from postmenopausal breast cancer, with the differences being limited to ER-positive tumors [44].

Whether the age-related biological differences within subtypes fully can explain the worse outcome for young women, or if treatment also plays a major role here, remains unclear. Except for age-related differences in the given treatment, one must also consider age-related differences in compliance to and effect of treatment. In the present study, all women were undertreated by today's standards, with chemotherapy given to only $76 \%$ of women $<35$ years and endocrine treatment to those with hormone receptor-positive disease in only $70 \%$. Ovarian suppression was offered to one-third of the youngest women with hormone receptor-positive tumors. Some authors have found young women to be less compliant with endocrine treatment [45-47]. Women with Luminal B breast cancer derive less benefit from endocrine therapy compared to those with Luminal A breast cancer [48], and likewise less benefit from paclitaxel and doxorubicin-containing preoperative chemotherapy compared with HER2enriched and basal-like breast cancers [49-51]. Studies on neoadjuvant chemotherapy in women with luminal tumors have shown women $<40$ years to have a higher rate of pathological complete response than women $>50$ years also with positive effect on survival [52]. However, survival differences between young and older women with luminal tumors have been demonstrated also in untreated cohorts [5, 39]. 

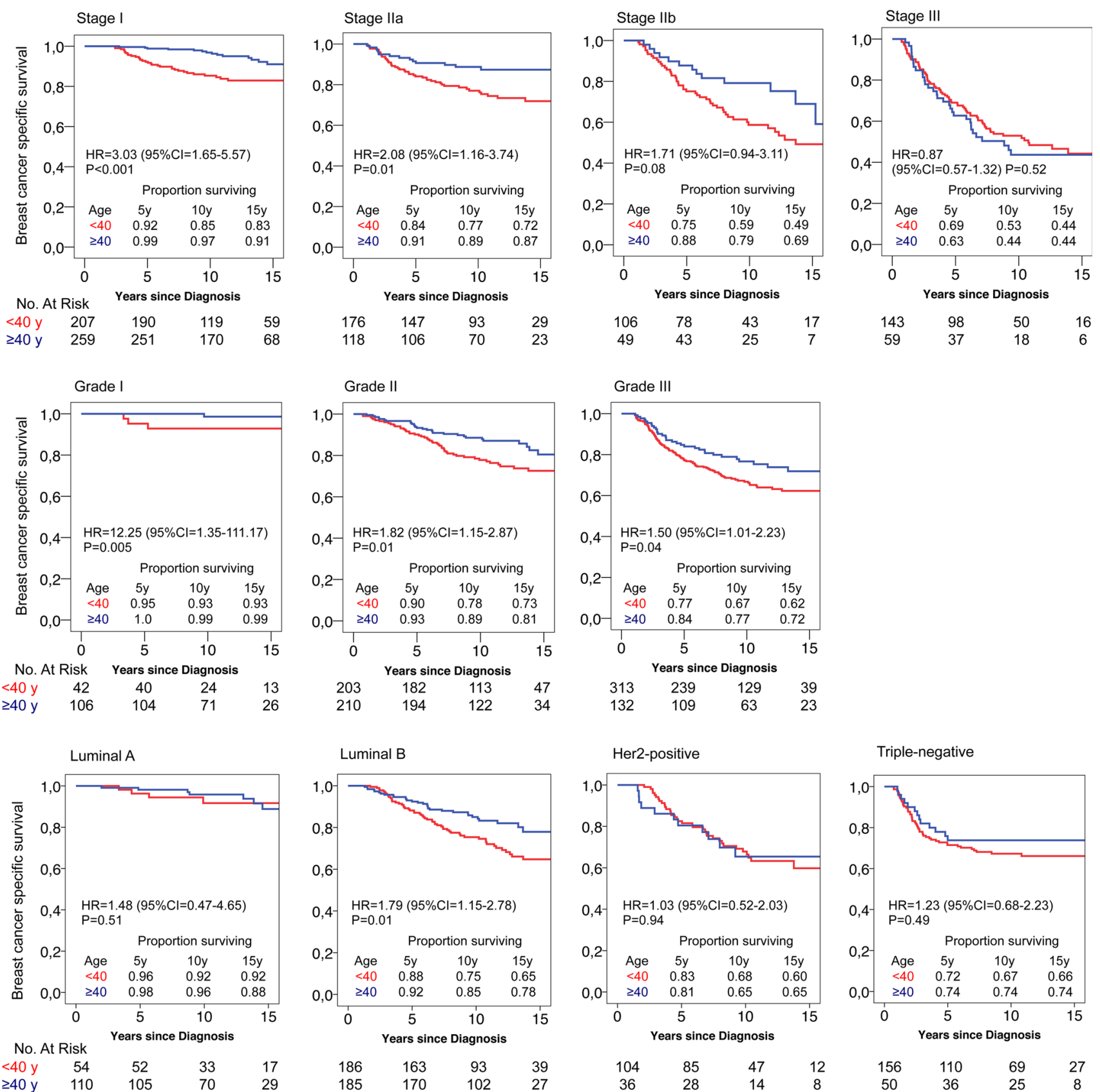

Fig. 2 Breast cancer-specific survival by age, stage, grade, and subtype for women with primary breast cancer stage I-III diagnosed $1992-2005(N=1120)$ divided by age $<40$ years and $\geq 40$ years.

To conclude, the effect of age is modified by tumor subtype. Despite correction for biology and more intense treatment in the young, young age is an independent risk factor for systemic disease in women with early-stage luminal tumors, with a two-fold risk of distant disease. However, current prognostic markers cannot reliably discriminate the young women benefitting from more intense systemic therapy and studies on prognostic markers
Hazard ratios (HR) are given with their $95 \%$ confidence intervals $(95 \% \mathrm{CI})$. Survival curves are compared by log-rank test. Proportion of women surviving at 5,10, and 15 years from diagnosis

relevant in the young population, and especially for the Luminal B subtype, are urgently needed. Age remains an important variable in treatment decisions until new relevant predictive markers are found.

Authors' Contributions Conception and design H. Fredholm, S. Eaker Fält, H. Lindman, L. Holmberg, J. Frisell, I. Fredriksson. 
Table 4 Multivariate analysis of prognostic factors ${ }^{\mathrm{a}}$ affecting breast cancer death, distant disease, and locoregional recurrence by age
Fig. 3 Forest plot of multivariate Cox regression of risk of event for women with stage I-IIa, estrogen receptor positive, and Her2-negative breast cancer $(N=389)$ by age $<40$ years $(n=152)$ versus reference $\geq 40$ years $(n=237)$. $B C S S$ Breast cancer-specific survival, DDFS distant diseasefree survival, LRFS locoregional recurrence-free survival. Open square Crude, filled square adjusted for diagnostic period, tumor size, lymph node status, grade, subtype (Luminal A or Luminal B), endocrine therapy, and chemotherapy

\begin{tabular}{|c|c|c|c|c|c|c|c|}
\hline & \multicolumn{2}{|c|}{$<35$ years } & \multicolumn{2}{|c|}{$35-39$ years } & \multicolumn{2}{|c|}{$40-49$ years } & \multirow{2}{*}{$\begin{array}{l}50-69 \text { years } \\
N=293\end{array}$} \\
\hline & $N=$ & & $N=$ & & $N=$ & & \\
\hline \multicolumn{8}{|l|}{ Breast cancer death } \\
\hline Unadjusted & 2.75 & (1.93-3.94) & 2.33 & $(1.54-3.52)$ & 1.53 & $(0.97-2.39)$ & 1.00 (ref) \\
\hline+ Year & 2.69 & (1.88-3.85) & 2.23 & $(1.48-3.38)$ & 1.45 & $(0.92-2.28)$ & \\
\hline+ Stage & 1.80 & $(1.25-2.60)$ & 1.42 & $(0.93-2.17)$ & 1.13 & $(0.72-1.78)$ & \\
\hline+ Detection mode & 1.39 & $(0.93-2.07)$ & 1.10 & $(0.70-1.72)$ & 0.94 & $(0.59-1.50)$ & \\
\hline +Grade & 1.16 & $(0.77-1.73)$ & 0.94 & $(0.59-1.49)$ & 0.85 & $(0.53-1.36)$ & \\
\hline +Subtype & 1.10 & $(0.73-1.64)$ & 0.93 & $(0.59-1.47)$ & 0.86 & $(0.53-1.38)$ & \\
\hline+ Systemic treatment & 1.04 & $(0.68-1.58)$ & 0.88 & $(0.55-1.41)$ & 0.84 & $(0.52-1.36)$ & \\
\hline \multicolumn{8}{|l|}{ Distant disease } \\
\hline Unadjusted & 3.11 & $(2.22-4.36)$ & 2.37 & $(1.60-3.53)$ & 1.74 & $(1.15-2.64)$ & 1.00 (ref) \\
\hline+ Year & 3.04 & $(2.17-4.26)$ & 2.28 & (1.53-3.39) & 1.65 & $(1.09-2.50)$ & \\
\hline+ Stage & 2.09 & (1.48-2.96) & 1.46 & $(0.97-2.18)$ & 1.29 & $(0.85-1.96)$ & \\
\hline+ Detection mode & 1.61 & (1.10-2.35) & 1.13 & $(0.73-1.73)$ & 1.07 & $(0.70-1.66)$ & \\
\hline +Grade & 1.41 & $(0.96-2.06)$ & 1.02 & $(0.66-1.57)$ & 1.00 & $(0.65-1.55)$ & \\
\hline +Subtype & 1.40 & $(0.96-2.05)$ & 1.01 & $(0.65-1.55)$ & 1.00 & $(0.65-1.55)$ & \\
\hline+ Systemic treatment & 1.36 & $(0.91-2.02)$ & 0.97 & $(0.62-1.52)$ & 0.99 & $(0.64-1.54)$ & \\
\hline \multicolumn{8}{|l|}{ Locoregional recurrence } \\
\hline Unadjusted & 3.16 & $(1.98-5.04)$ & 2.88 & $(1.70-4.89)$ & 1.94 & $(1.11-3.41)$ & 1.00 (ref) \\
\hline+ Year & 3.09 & $(1.94-4.94)$ & 2.80 & $(1.65-4.78)$ & 1.85 & $(1.05-3.25)$ & \\
\hline+ Stage & 2.88 & $(1.79-4.64)$ & 2.60 & $(1.52-4.45)$ & 1.78 & $(1.01-3.15)$ & \\
\hline+ Detection mode & 2.38 & $(1.37-4.12)$ & 2.15 & $(1.18-3.93)$ & 1.58 & $(0.87-2.86)$ & \\
\hline +Grade & 2.11 & $(1.21-3.67)$ & 1.96 & $(1.07-3.59)$ & 1.50 & $(0.82-2.72)$ & \\
\hline +Subtype & 2.09 & $(1.20-3.65)$ & 1.94 & $(1.06-3.57)$ & 1.51 & $(0.83-2.74)$ & \\
\hline+ Systemic treatment & 2.13 & $(1.21-3.76)$ & 1.97 & $(1.06-3.68)$ & 1.51 & $(0.83-2.75)$ & \\
\hline
\end{tabular}

Women with stage I-III breast cancer diagnosed 1992-2005 $(N=1120)$. Women age 50-69 serves as reference category. Hazard ratio (95\% confidence interval)

Bold values indicate statistical significance at the $p<0.05$ level

a Adjusted for year of diagnosis (1992-1997, 1998-2002, 2003-2005), stage (tumor size 1-10, 11-20, $\geq 20 \mathrm{~mm}$, missing and lymph node status; node neg, node pos), detection mode (screening or clinically detected), grade (Elston I, II, III, missing), subtype (Lum A, Lum B, Lum-Her2, Her2-pos, Triple-neg, unclassified), systemic treatment (chemotherapy and endocrine treatment including ovarian suppression)

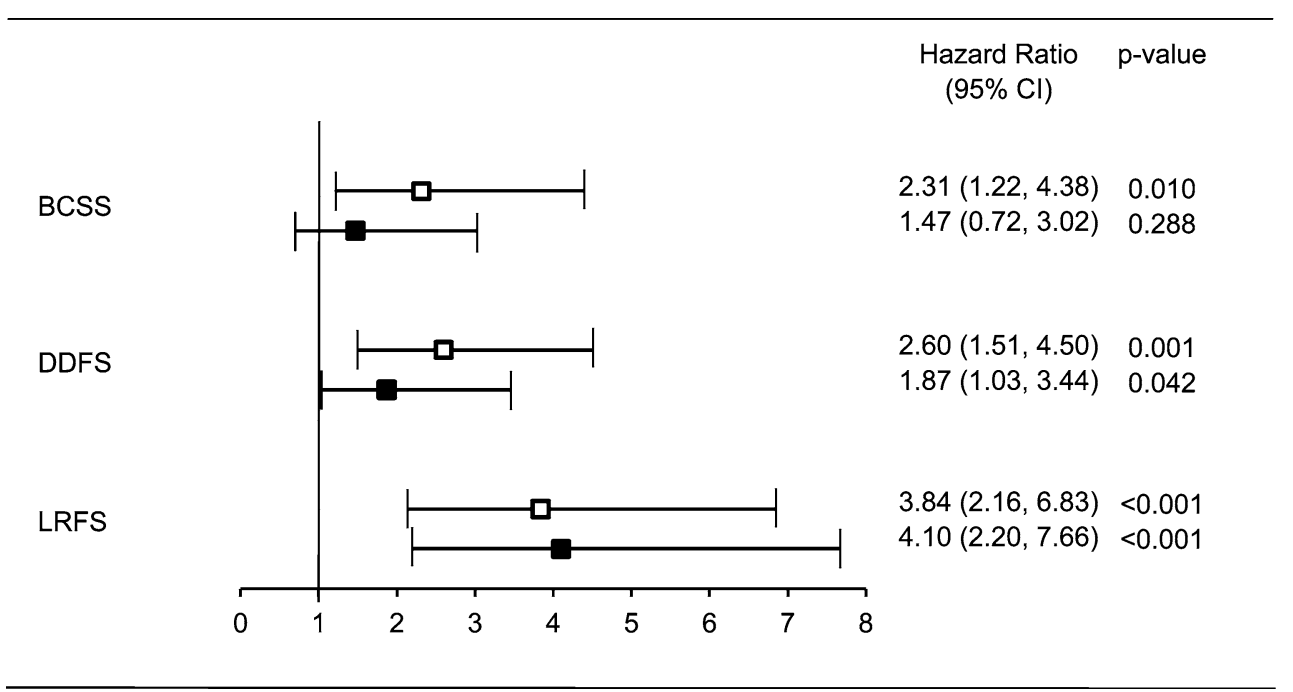


Development of methodology H. Fredholm, K. Magnusson, L. S. Lindström, H. Garmo, S. Eaker Fält, H. Lindman, J. Bergh, L. Holmberg, F. Pontén, J. Frisell, I. Fredriksson. Acquisition of data (provided animals, acquired and managed patients, provided facilities, etc.) H. Fredholm, K. Magnusson, L. S Lindström, H. Garmo, S. Eaker Fält, H. Lindman, J. Bergh, L. Holmberg, F. Pontén, J. Frisell, I. Fredriksson. Analysis and interpretation of data (e.g., statistical analysis, biostatistics, computational analysis) H. Fredholm, K. Magnusson, L. S. Lindström, H. Garmo, S. Eaker Fält, H. Lindman, J. Bergh, L. Holmberg, F. Pontén, J. Frisell, I. Fredriksson. Writing, review, and/or revision of the manuscript $\mathrm{H}$. Fredholm, K. Magnusson, L. S. Lindström, H. Garmo, S. Eaker Fält, H. Lindman, J. Bergh, L. Holmberg, F. Pontén, J. Frisell, I. Fredriksson. Administrative, technical, or material support (i.e., reporting or organizing data, constructing databases) H. Fredholm, K. Magnusson, L. S. Lindström, H. Garmo, S. Eaker Fält, H. Lindman, J. Bergh, L. Holmberg, F. Pontén, J. Frisell, I. Fredriksson. Study supervision I. Fredriksson.

Funding This work was financially supported through the regional agreement on medical training and clinical research (ALF) between the Stockholm County Council and Karolinska Institutet (Fredholm, Frisell, Fredriksson, Bergh), by the Swedish Breast Cancer Association (BRO)(Fredholm, Fredriksson), the BRECT Theme Network (Fredholm, Fredriksson, Bergh), by the Swedish Cancer Society (Fredholm, Frisell, Fredriksson, Pontén, Bergh), by the Swedish Research Council (Lindström (grant no 524-2011-6857)), the Cancer Research Foundations of Radiumhemmet (Bergh) and the Knut and Alice Wallenberg Foundation (Pontén, Magnusson).

\section{Compliance with Ethical Standards}

Conflicts of Interest The authors declare that they have no conflicts of interest.

Ethical approval All procedures performed in this study involving human participants were in accordance with the ethical standards of the Research Ethics Committee at Karolinska Institutet, Stockholm, Sweden (approval diary number 2009/1174-31/1 and 2010/586-32) and with the 1964 Helsinki declaration and its later amendments or comparable ethical standards.

Informed consent The Research Ethics Committee at Karolinska Institutet, Stockholm, Sweden, approved an informed consent waiver for the retrospective medical record review (approval diary number 2009/1174-31/1 and 2010/586-32).

Open Access This article is distributed under the terms of the Creative Commons Attribution 4.0 International License (http://crea tivecommons.org/licenses/by/4.0/), which permits unrestricted use, distribution, and reproduction in any medium, provided you give appropriate credit to the original author(s) and the source, provide a link to the Creative Commons license, and indicate if changes were made.

\section{References}

1. Kroman N, Jensen MB, Wohlfahrt J, Mouridsen HT, Andersen PK, Melbye M (2000) Factors influencing the effect of age on prognosis in breast cancer: population based study. BMJ 320(7233):474-478

2. Bharat A, Aft RL, Gao F, Margenthaler JA (2009) Patient and tumor characteristics associated with increased mortality in young women $(<$ or $=40$ years $)$ with breast cancer. J Surg Oncol 100(3):248-251. doi:10.1002/jso.21268
3. Fredholm H, Eaker S, Frisell J, Holmberg L, Fredriksson I, Lindman H (2009) Breast cancer in young women: poor survival despite intensive treatment. Plos One 4(11):e7695. doi:10.1371/ journal.pone. 0007695

4. Maggard MA, O'Connell JB, Lane KE, Liu JH, Etzioni DA, Ko CY (2003) Do young breast cancer patients have worse outcomes? J Surg Res 113(1):109-113. doi:10.1016/S00224804(03)00179-3

5. Azim HA Jr, Michiels S, Bedard PL, Singhal SK, Criscitiello C, Ignatiadis M, Haibe-Kains B, Piccart MJ, Sotiriou C, Loi S (2012) Elucidating prognosis and biology of breast cancer arising in young women using gene expression profiling. Clin Cancer Res 18(5):1341-1351. doi:10.1158/1078-0432.CCR-11-2599

6. Gnerlich JL, Deshpande AD, Jeffe DB, Sweet A, White N, Margenthaler JA (2009) Elevated breast cancer mortality in women younger than age 40 years compared with older women is attributed to poorer survival in early-stage disease. J Am Coll Surg 208(3):341-347. doi:10.1016/j.jamcollsurg.2008.12.001

7. Adami HO, Malker B, Holmberg L, Persson I, Stone B (1986) The relation between survival and age at diagnosis in breast cancer. N Engl J Med 315(9):559-563. doi:10.1056/ NEJM198608283150906

8. Partridge AH, Hughes ME, Ottesen RA, Wong YN, Edge SB, Theriault RL, Blayney DW, Niland JC, Winer EP, Weeks JC, Tamimi RM (2012) The effect of age on delay in diagnosis and stage of breast cancer. Oncologist 17(6):775-782. doi:10.1634/ theoncologist.2011-0469

9. Colleoni M, Rotmensz N, Robertson C, Orlando L, Viale G, Renne G, Luini A, Veronesi P, Intra M, Orecchia R, Catalano G, Galimberti V, Nole F, Martinelli G, Goldhirsch A (2002) Very young women $(<35$ years) with operable breast cancer: features of disease at presentation. Ann Oncol 13(2):273-279

10. Anders CK, Hsu DS, Broadwater G, Acharya CR, Foekens JA, Zhang Y, Wang Y, Marcom PK, Marks JR, Febbo PG, Nevins JR, Potti A, Blackwell KL (2008) Young age at diagnosis correlates with worse prognosis and defines a subset of breast cancers with shared patterns of gene expression. J Clin Oncol 26(20):3324-3330. doi:10.1200/JCO.2007.14.2471

11. Lund MJ, Butler EN, Hair BY, Ward KC, Andrews JH, OpreaIlies G, Bayakly AR, O'Regan RM, Vertino PM, Eley JW (2010) Age/race differences in HER2 testing and in incidence rates for breast cancer triple subtypes: a population-based study and first report. Cancer 116(11):2549-2559. doi:10.1002/cncr.25016

12. Tang LC, Jin X, Yang HY, He M, Chang H, Shao ZM, Di GH (2015) Luminal B subtype: a key factor for the worse prognosis of young breast cancer patients in China. BMC Cancer 15:201. doi:10.1186/s12885-015-1207-z

13. Morrison DH, Rahardja D, King E, Peng Y, Sarode VR (2012) Tumour biomarker expression relative to age and molecular subtypes of invasive breast cancer. Br J Cancer 107(2):382-387. doi:10.1038/bjc.2012.219

14. Sorlie T, Perou CM, Tibshirani R, Aas T, Geisler S, Johnsen H, Hastie T, Eisen MB, van de Rijn M, Jeffrey SS, Thorsen T, Quist $\mathrm{H}$, Matese JC, Brown PO, Botstein D, Lonning PE, BorresenDale AL (2001) Gene expression patterns of breast carcinomas distinguish tumor subclasses with clinical implications. Proc Natl Acad Sci USA 98(19):10869-10874. doi:10.1073/pnas.19136 7098

15. Cancello G, Maisonneuve P, Rotmensz N, Viale G, Mastropasqua MG, Pruneri G, Veronesi P, Torrisi R, Montagna E, Luini A, Intra M, Gentilini O, Ghisini R, Goldhirsch A, Colleoni M (2010) Prognosis and adjuvant treatment effects in selected breast cancer subtypes of very young women ( $<35$ years) with operable breast cancer. Ann Oncol 21(10):1974-1981. doi:10.1093/annonc/ mdq072 
16. Liedtke C, Hess KR, Karn T, Rody A, Kiesel L, Hortobagyi GN, Pusztai L, Gonzalez-Angulo AM (2013) The prognostic impact of age in patients with triple-negative breast cancer. Breast Cancer Res Treat 138(2):591-599. doi:10.1007/s10549-013-2461-x

17. Blows FM, Driver KE, Schmidt MK, Broeks A, van Leeuwen FE, Wesseling J, Cheang MC, Gelmon K, Nielsen TO, Blomqvist C, Heikkila P, Heikkinen T, Nevanlinna H, Akslen LA, Begin LR, Foulkes WD, Couch FJ, Wang X, Cafourek V, Olson JE, Baglietto L, Giles GG, Severi G, McLean CA, Southey MC, Rakha E, Green AR, Ellis IO, Sherman ME, Lissowska J, Anderson WF, Cox A, Cross SS, Reed MW, Provenzano E, Dawson SJ, Dunning AM, Humphreys M, Easton DF, GarciaClosas M, Caldas C, Pharoah PD, Huntsman D (2010) Subtyping of breast cancer by immunohistochemistry to investigate a relationship between subtype and short and long term survival: a collaborative analysis of data for 10,159 cases from 12 studies. Plos Med 7(5):e1000279. doi:10.1371/journal.pmed.1000279

18. Sheridan W, Scott T, Caroline S, Yvonne Z, Vanessa B, David V, Karen G, Stephen C (2014) Breast cancer in young women: have the prognostic implications of breast cancer subtypes changed over time? Breast Cancer Res Treat 147(3):617-629. doi:10. 1007/s10549-014-3125-1

19. Edge SB, Compton CC (2010) The American Joint Committee on Cancer: the 7th edition of the AJCC cancer staging manual and the future of TNM. Ann Surg Oncol 17(6):1471-1474. doi:10. 1245/s10434-010-0985-4

20. von Elm E, Altman DG, Egger M, Pocock SJ, Gotzsche PC, Vandenbroucke JP, Initiative S (2007) The strengthening the reporting of observational studies in epidemiology (STROBE) statement: guidelines for reporting observational studies. Lancet 370(9596):1453-1457. doi:10.1016/S0140-6736(07)61602-X

21. McShane LM, Altman DG, Sauerbrei W, Taube SE, Gion M, Clark GM, Statistics Subcommittee of the NCIEWGoCD (2005) Reporting recommendations for tumor marker prognostic studies (REMARK). J Natl Cancer Inst 97(16):1180-1184. doi:10.1093/ jnci/dji237

22. Elston CW, Ellis IO (1991) Pathological prognostic factors in breast cancer. I. The value of histological grade in breast cancer: experience from a large study with long-term follow-up. Histopathology 19(5):403-410

23. Uhlen M, Oksvold P, Fagerberg L, Lundberg E, Jonasson K, Forsberg M, Zwahlen M, Kampf C, Wester K, Hober S, Wernerus H, Bjorling L, Ponten F (2010) Towards a knowledge-based Human Protein Atlas. Nat Biotechnol 28(12):1248-1250. doi:10. 1038/nbt1210-1248

24. Ponten F, Schwenk JM, Asplund A, Edqvist PH (2011) The Human Protein Atlas as a proteomic resource for biomarker discovery. J Intern Med 270(5):428-446. doi:10.1111/j.13652796.2011.02427.x

25. Kampf C, Olsson I, Ryberg U, Sjostedt E, Ponten F (2012) Production of tissue microarrays, immunohistochemistry staining and digitalization within the human protein atlas. J Vis Exp. doi: $10.3791 / 3620$

26. Dowsett M, Nielsen TO, A'Hern R, Bartlett J, Coombes RC, Cuzick J, Ellis M, Henry NL, Hugh JC, Lively T, McShane L, Paik S, Penault-Llorca F, Prudkin L, Regan M, Salter J, Sotiriou C, Smith IE, Viale G, Zujewski JA, Hayes DF (2011) Assessment of Ki67 in breast cancer: recommendations from the International Ki67 in Breast Cancer working group. J Natl Cancer Inst 103(22):1656-1664. doi:10.1093/jnci/djr393

27. Goldhirsch A, Winer EP, Coates AS, Gelber RD, Piccart-Gebhart M, Thurlimann B, Senn HJ, Panel M (2013) Personalizing the treatment of women with early breast cancer: highlights of the St Gallen International Expert Consensus on the primary therapy of early breast cancer 2013. Ann Oncol 24(9):2206-2223. doi:10. 1093/annonc/mdt303
28. Wolff AC, Hammond ME, Schwartz JN, Hagerty KL, Allred DC, Cote RJ, Dowsett M, Fitzgibbons PL, Hanna WM, Langer A, McShane LM, Paik S, Pegram MD, Perez EA, Press MF, Rhodes A, Sturgeon C, Taube SE, Tubbs R, Vance GH, van de Vijver M, Wheeler TM, Hayes DF, American Society of Clinical Oncology, College of American Pathologists (2007) American Society of Clinical Oncology/College of American Pathologists guideline recommendations for human epidermal growth factor receptor 2 testing in breast cancer. J Clin Oncol 25(1):118-145. doi:10. 1200/JCO.2006.09.2775

29. Tanner M, Gancberg D, Di Leo A, Larsimont D, Rouas G, Piccart MJ, Isola J (2000) Chromogenic in situ hybridization: a practical alternative for fluorescence in situ hybridization to detect HER-2/ neu oncogene amplification in archival breast cancer samples. Am J Pathol 157(5):1467-1472. doi:10.1016/S0002-9440(10) 64785-2

30. van de Vijver M, Bilous M, Hanna W, Hofmann M, Kristel P, Penault-Llorca F, Ruschoff J (2007) Chromogenic in situ hybridisation for the assessment of HER2 status in breast cancer: an international validation ring study. Breast Cancer Res 9(5):R68. doi:10.1186/bcr1776

31. Kaplan EL, Meier P (1958) Nonparametric estimation from incomplete observations. J Am Stat Assoc 53:457-481

32. Mantel N (1966) Evaluation of survival data and two new rank order statistics arising in its consideration. Cancer Chemother Rep 50(3):163-170

33. Cox DR (1972) Regression models and life-tables. J R Stat Soc B34:187-220

34. Yersal O, Barutca S (2014) Biological subtypes of breast cancer: prognostic and therapeutic implications. World J Clin Oncol 5(3):412-424. doi:10.5306/wjco.v5.i3.412

35. Ademuyiwa FO, Gao F, Hao L, Morgensztern D, Aft RL, Ma CX, Ellis MJ (2015) US breast cancer mortality trends in young women according to race. Cancer 121(9):1469-1476. doi:10. $1002 /$ cncr. 29178

36. Kim EK, Noh WC, Han W, Noh DY (2011) Prognostic significance of young age $(<35$ years) by subtype based on ER, PR, and HER2 status in breast cancer: a nationwide registry-based study. World J Surg 35(6):1244-1253. doi:10.1007/s00268-011-1071-1

37. Goldhirsch A, Gelber RD, Yothers G, Gray RJ, Green S, Bryant J, Gelber S, Castiglione-Gertsch M, Coates AS (2001) Adjuvant therapy for very young women with breast cancer: need for tailored treatments. J Natl Cancer Inst Monogr 30:44-51

38. Colleoni M, Rotmensz N, Peruzzotti G, Maisonneuve P, Orlando L, Ghisini R, Viale G, Pruneri G, Veronesi P, Luini A, Intra M, Cardillo A, Torrisi R, Rocca A, Goldhirsch A (2006) Role of endocrine responsiveness and adjuvant therapy in very young women (below 35 years) with operable breast cancer and node negative disease. Ann Oncol 17(10):1497-1503. doi:10.1093/ annonc/mdl145

39. Ahn SH, Son BH, Kim SW, Kim SI, Jeong J, Ko SS, Han W, Korean Breast Cancer Society (2007) Poor outcome of hormone receptor-positive breast cancer at very young age is due to tamoxifen resistance: nationwide survival data in Korea-a report from the Korean Breast Cancer Society. J Clin Oncol 25(17):2360-2368. doi:10.1200/JCO.2006.10.3754

40. Aebi S, Gelber S, Castiglione-Gertsch M, Gelber RD, Collins J, Thurlimann B, Rudenstam CM, Lindtner J, Crivellari D, CortesFunes H, Simoncini E, Werner ID, Coates AS, Goldhirsch A (2000) Is chemotherapy alone adequate for young women with oestrogen-receptor-positive breast cancer? Lancet 355(9218): 1869-1874

41. Lin $\mathrm{CH}$, Chuang PY, Chiang CJ, Lu YS, Cheng AL, Kuo WH, Huang CS, Lai MS, You SL, Tang CH (2014) Distinct clinicopathological features and prognosis of emerging young-female breast cancer in an East Asian country: a nationwide cancer 
registry-based study. Oncologist 19(6):583-591. doi:10.1634/ theoncologist.2014-0047

42. Park YH, Lee SJ, Jung HA, Kim SM, Kim MJ, Kil WH, Lee JE, Nam SJ, Ahn JS, Im YH (2015) Prevalence and clinical outcomes of young breast cancer (YBC) patients according to intrinsic breast cancer subtypes: single institutional experience in Korea. Breast 24(3):213-217. doi:10.1016/j.breast.2015.01.012

43. Johnson RH, Hu P, Fan C, Anders CK (2015) Gene expression in "young adult type" breast cancer: a retrospective analysis. Oncotarget 6(15):13688-13702

44. Liao S, Hartmaier RJ, McGuire KP, Puhalla SL, Luthra S, Chandran UR, Ma T, Bhargava R, Modugno F, Davidson NE, Benz S, Lee AV, Tseng GC, Oesterreich S (2015) The molecular landscape of premenopausal breast cancer. Breast Cancer Res 17:104. doi:10.1186/s13058-015-0618-8

45. Hershman DL, Kushi LH, Shao T, Buono D, Kershenbaum A, Tsai WY, Fehrenbacher L, Gomez SL, Miles S, Neugut AI (2010) Early discontinuation and nonadherence to adjuvant hormonal therapy in a cohort of 8,769 early-stage breast cancer patients. J Clin Oncol 28(27):4120-4128. doi:10.1200/JCO.2009.25.9655

46. Partridge AH, Wang PS, Winer EP, Avorn J (2003) Nonadherence to adjuvant tamoxifen therapy in women with primary breast cancer. J Clin Oncol 21(4):602-606

47. He W, Fang F, Varnum C, Eriksson M, Hall P, Czene K (2015) Predictors of discontinuation of adjuvant hormone therapy in patients with breast cancer. J Clin Oncol 33(20):2262-2269. doi:10.1200/JCO.2014.59.3673

48. Ades F, Zardavas D, Bozovic-Spasojevic I, Pugliano L, Fumagalli D, de Azambuja E, Viale G, Sotiriou C, Piccart M (2014) Luminal B breast cancer: molecular characterization, clinical management, and future perspectives. J Clin Oncol 32(25):2794-2803. doi:10. 1200/JCO.2013.54.1870
49. Bhargava R, Beriwal S, Dabbs DJ, Ozbek U, Soran A, Johnson RR, Brufsky AM, Lembersky BC, Ahrendt GM (2010) Immunohistochemical surrogate markers of breast cancer molecular classes predicts response to neoadjuvant chemotherapy: a single institutional experience with 359 cases. Cancer 116(6):1431-1439. doi:10.1002/cncr.24876

50. Carey LA, Dees EC, Sawyer L, Gatti L, Moore DT, Collichio F, Ollila DW, Sartor CI, Graham ML, Perou CM (2007) The triple negative paradox: primary tumor chemosensitivity of breast cancer subtypes. Clin Cancer Res 13(8):2329-2334. doi:10.1158/ 1078-0432.CCR-06-1109

51. Esserman LJ, Berry DA, Cheang MC, Yau C, Perou CM, Carey L, DeMichele A, Gray JW, Conway-Dorsey K, Lenburg ME, Buxton MB, Davis SE, van't Veer LJ, Hudis C, Chin K, Wolf D, Krontiras H, Montgomery L, Tripathy D, Lehman C, Liu MC, Olopade OI, Rugo HS, Carpenter JT, Livasy C, Dressler L, Chhieng D, Singh B, Mies C, Rabban J, Chen YY, Giri D, Au A, Hylton N, Investigators IST (2012) Chemotherapy response and recurrence-free survival in neoadjuvant breast cancer depends on biomarker profiles: results from the I-SPY 1 TRIAL (CALGB 150007/150012; ACRIN 6657). Breast Cancer Res Treat 132(3):1049-1062. doi:10.1007/s10549-011-1895-2

52. Loibl S, Jackisch C, Lederer B, Untch M, Paepke S, Kummel S, Schneeweiss A, Huober J, Hilfrich J, Hanusch C, Gerber B, Eidtmann H, Denkert C, Costa SD, Blohmer JU, Nekljudova V, Mehta K, von Minckwitz G (2015) Outcome after neoadjuvant chemotherapy in young breast cancer patients: a pooled analysis of individual patient data from eight prospectively randomized controlled trials. Breast Cancer Res Treat 152(2):377-387. doi:10.1007/s10549-015-3479-z 